\title{
Effects of edaravone on the expression of $\beta$-defensin-2 mRNA in lung tissue of rats with myocardial ischemia reperfusion
}

\author{
WEIWEI ZHANG, YONGQING GUO, SHUZHEN YU, JIANFENG WEI and JIANMIN JIN \\ Department of Anesthesiology, Shanxi Provincial People's Hospital, Taiyuan, Shanxi 030012, P.R. China
}

Received November 22, 2012; Accepted March 1, 2013

DOI: $10.3892 / \mathrm{mmr} .2013 .1393$

\begin{abstract}
The aim of this study was to investigate the effects of edaravone on lung injury caused by myocardial ischemia reperfusion $(\mathrm{I} / \mathrm{R})$ in rats. Wistar rats $(\mathrm{n}=24)$ were randomly divided into 4 groups: the sham operation (S group) and myocardial I/R groups (C group) and two edaravone-treated groups $\left(E_{1}\right.$ and $E_{2}$ groups). Rats in the $E_{1}$ and $E_{2}$ groups were injected with 3 or $10 \mathrm{mg} / \mathrm{kg}$ edaravone, respectively, $1 \mathrm{~min}$ before reperfusion. The rats were sacrificed and the lung tissue, bronchoalveolar lavage (BAL) fluid and serum were obtained. The concentration of serum creatine kinase isoenzyme (CK-MB) was determined, the lung permeability index (PPI) was calculated and $\beta$-defensin-2 (BD-2) mRNA expression in the lung tissue and BD-2 and TNF- $\alpha$ protein content levels were determined. Serum CK-MB activity and the PPI were increased, while BD-2 mRNA and BD-2 and TNF- $\alpha$ protein levels in the lung tissue were upregulated in the $\mathrm{C}, \mathrm{E}_{1}$ and $\mathrm{E}_{2}$ groups compared with the $\mathrm{S}$ group. The above-mentioned indicators were decreased in the $\mathrm{E}_{1}$ and $\mathrm{E}_{2}$ groups compared with the IR group. The level of the decrease for indicators in the $\mathrm{E}_{2}$ group was significantly different compared with that in the $\mathrm{E}_{1}$ group. In conclusion, edaravone reduced the lung injury caused by myocardial $I / R$ in rats. Its mechanism of action was not only oxygen free radical scavenging, but was also associated with a suppression of the inflammatory response of the lung tissue.
\end{abstract}

\section{Introduction}

Myocardial ischemia reperfusion (ischemia/reperfusion, I/R) injury significantly impacts the recovery of heart function following ischemia and may even be life-threatening. Consequently, the effective prevention and treatment of myocardial I/R injury has become the focus of numerous studies. Murry et al (1) first proposed the myocardial ischemic preconditioning process in 1986. Following this, ischemic

Correspondence to: Professor Yongqing Guo, Department of Anesthesiology, Shanxi Provincial People's Hospital, No. 29 Shuangtasi Street, Yingze, Taiyuan, Shanxi 030012, P.R. China E-mail: gyq106968@yahoo.com.cn

Key words: radical scavenger, myocardial reperfusion injury, respiratory distress syndrome, adult post-processing $(2,3)$ and remote ischemic post-processing (4) were suggested to be effective against myocardial I/R injury and play a role in myocardial protection.

Previous studies have demonstrated that I/R of the heart causes damage to the lungs and has a significant impact on the treatment and prognosis of patients. Kitashiro et al (5) carried out in vivo experiments in dogs which showed that myocardial I/R injury significantly increased lung water content. Tang et al (4) demonstrated that the remote postconditioning induced by brief occlusion and reperfusion of the pulmonary artery could attenuate myocardial reperfusion injury. The left pulmonary artery was blocked for $5 \mathrm{~min}$ followed by a 5 -min reperfusion, and the left anterior descending coronary artery was occlude for 30 min with a $180-$ min reperfusion. Furthermore, the mechanism of protection was associated with the activation of endothelial nitric oxide synthase (eNOS). A study by $\mathrm{He}$ et al (6) revealed that the application of anti-human tissue factor antibodies may play a protective role in intestinal I/R-induced lung injury. An increased concentration of proinflammatory cytokines has been identified in the plasma of acute kidney injury patients, which leads to an increased incidence of respiratory complications (7). Following acute kidney injury in rats, the lungs exhibited an increase in pulmonary capillary leakage and lung myeloperoxidase activity (8), suggesting that other remote organs with I/R may also cause lung complications. Therefore, the occurrence and development of lung injury and protective measures require attention. Lung injury following myocardial $\mathrm{I} / \mathrm{R}$ is mainly characterized by a significant accumulation of polymorphonuclear leukocytes, which produce large numbers of cytokines and oxygen free radicals that attack alveolar cell mitochondria, leading to increased pulmonary capillary permeability and the development of lung injury (5).

$\beta$-defensin-2 (BD-2) is a small cationic antimicrobial peptide. Human BD-2 was initially identified in epithelial tissue and the lungs and is secreted following stimulation by bacterial infections and inflammation. In a previous study recombinant adenovirus carrying an expression cassette of rat BD-2 was administered intratracheally to Sprague-Dawley rats $48 \mathrm{~h}$ prior to performing acute lung injury, which was induced by Pseudomonas aeruginosa infection. The level of $P$. aeruginosa in the lung with BD-2 overexpression declined significantly. Overexpression of BD-2 reduced alveolar damage, interstitial edema and infiltration of neutrophils in the model. Furthermore, Pseudomonas aeruginosa infection in the lungs of cystic fibrosis patients has been shown to be strongly associ- 
ated with the inactivation of BD-2 (9). Hu et al (10) developed a rat model that excessively expressed human BD-2 through gene regulation, which significantly prolonged the survival time of rats following infection with Pseudomonas aerugi$n o s a$. This also reduced the aggregation of bacteria in the lungs following infection and regulated the expression of numerous types of cells/inflammatory cytokines during the early stages of inflammation (including interleukin-1 $\beta$ and TNF- $\alpha$ ). BD-2 may be able to directly kill microorganisms in the lung tissue and regulate the immune process. Wu et al (11) showed that acute lung injury and inflammation of the lungs induced by different modes of mechanical ventilation affect BD-2 expression. A number of studies have demonstrated that the BD-2 gene in lung tissue is upregulated following intestinal $\mathrm{I} / \mathrm{R}$ in rats (12). However, no previous studies have investigated the changes in BD-2 expression in lung tissue following myocardial I/R. Edaravone is a novel free radical scavenger, which inhibits reactive oxygen species generated by $\mathrm{I} / \mathrm{R}$ and thus has a protective effect in heart, brain and liver damage (13-15). However, the effects of edaravone on myocardial I/R-induced lung injury have not been investigated.

Therefore, the aims of the present study were to investigate BD-2 expression in the lung tissue following myocardial I/R, observe the protective effects of edaravone in lung injury following heart $\mathrm{I} / \mathrm{R}$ processing and discuss whether this may have an effect on BD-2 expression in lung tissue following myocardial ischemia.

\section{Materials and methods}

Materials. A one-step total RNA extraction kit and DNA polymerase were purchased from Beijing TransGen Biotech Co., Ltd. (Beijing, China). RNA enzyme inhibitors, murine leukemia virus reverse transcriptase, deoxynucleotide triphosphates (dNTPs) and a direct cloning carrier of PCR products vector system were from Fermentas (Toronto, ON, Canada). Mouse-derived $\beta$-actin (sc-47778), sheep-derived TNF- $\alpha$ (sc-1348) and rabbit-derived $\beta$-defensin antibodies (bs-1296R) were purchased from Beijing Biosynthesis Biotechnology Co., Ltd. (Beijing, China).

Animals. Healthy and clean male Wistar rats (weight, 250-300 g; $\mathrm{n}=24$ ) were provided by the Department of Physiology of Shanxi Medical Laboratory Animal Center (Taiyuan, China). The rats were randomly divided into 4 groups (n=6/group); the sham operation ( $\mathrm{S}$ group), myocardial I/R control (C group) and edaravone-treated groups $\left(\mathrm{E}_{1}\right.$ and $\mathrm{E}_{2}$ groups; different doses). The study was approved by the ethics committee of Shanxi Provincial People's Hospital, Taiyuan, China.

Construction of the rat model. The myocardial I/R model was constructed according to previously described methods (16). The rats were weighed and anesthetized with an intraperitoneal injection of urethane $(25 \% ; 3 \mathrm{mg} / \mathrm{kg})$. A HX-300 animal ventilator was used for breathing assistance following tracheal cannulation and the standard limb lead ECG was traced. A thoracotomy was performed to expose the heart, and the left anterior descending branch (LAD) was separated between the left atrial appendage and pulmonary cone. A 7/0 non-invasive suture was used through the myocardium surface, $2 \mathrm{~mm}$ below the root of the left atrial appendage, and the needle was withdrawn beside the pulmonary conus. The electrocardiogram was observed for $15 \mathrm{~min}$ to allow the heart to become stable, then the LAD was ligated. Elevation of the ST-segment in lead-II and the darkening of the corresponding region on the surface of the heart indicated the successful induction of ischemia. The ligature was untied after $45 \mathrm{~min}$. When the ECG ST-segment had declined $>1 / 3$ and the surface of the heart was recovered to a red color, this indicated that the myocardium had recovered from the reperfusion and further reperfusion was continued for $3 \mathrm{~h}$. The rats in the $\mathrm{E}_{1}$ and $\mathrm{E}_{2}$ groups were intravenously injected with 3 or $10 \mathrm{mg} / \mathrm{kg}$ edaravone (2010031; Boda Pharmaceutical Co., Ltd., Jilin, China), respectively, 1 min before reperfusion via the right femoral vein and reperfusion was performed for an additional $3 \mathrm{~h}$. The myocardial I/R rat model for the $\mathrm{C}$ group was constructed according to the method described above, while threading without ligation was performed in rats of the $\mathrm{S}$ group.

Sample collection. After $3 \mathrm{~h}$ of reperfusion, the rats were sacrificed using rapid abdominal aorta phlebotomy. Arterial blood samples $(5 \mathrm{ml})$ were obtained by bloodletting, allowed to stand at room temperature for $60 \mathrm{~min}$ and centrifuged at $12,000 \mathrm{x} g$ for $10 \mathrm{~min}$. The supernatant was allowed to stand for a further $30 \mathrm{~min}$ and centrifuged at $12,000 \mathrm{x} \mathrm{g}$ for $10 \mathrm{~min}$. The resulting supernatant was loaded into Eppendorf tubes, which were sealed and placed in a $-70^{\circ} \mathrm{C}$ refrigerator. Subsequently, serum CK-MB activity was detected using the CCX automatic biochemical analyzer (Abbott, Abbott Park, IL, USA); the kit was provided by Boehringer (Mannheim, Germany).

After the rats were sacrificed, the sternum was dissected along the midline to expose the lungs. A curved hemostat was used to block the right lung hilum and a V-shaped incision tracheostomy was made at the bottom of the tracheotomy spot. A sterile infusion tube (inner diameter, $\sim 2 \mathrm{~mm}$ ) was inserted through this incision into the left bronchus and the sterile thin tube was fixed above the carina using suture ligation. Sterile saline $(2 \mathrm{ml})$ was fractionally injected through the thin tube using a syringe five times and the left lung was lavaged with $20 \mathrm{~cm} \mathrm{H}_{2} \mathrm{O}$ pressure $\left(1 \mathrm{~cm} \mathrm{H}_{2} \mathrm{O}=0.098 \mathrm{kPa}\right)$. The lavage fluid was drawn back into the syringe and this was slowly repeated 4-5 times; the lavage fluid was collected in dry and sterile glass tubes. The duration of the complete lung lavage process was $\sim 20 \mathrm{~min}$. The lavage fluid was maintained at room temperature for $30 \mathrm{~min}$ and centrifuged at $10,000 \mathrm{x} \mathrm{g}$ for $10 \mathrm{~min}$. The supernatant was sealed in an Eppendorf tube and stored in a $-70^{\circ} \mathrm{C}$ refrigerator. The Coomassie brilliant blue staining method was used for protein quantification and the lavage fluid protein contents were determined according to the manufacturer's instructions (Nanjing Jiancheng Bioengineering Institute, Nanjing, China). The obtained data were used to determine the lung permeability index (PPI), the protein concentration in the lavage fluid and the serum protein concentration ratio.

BD-2 and TNF- $\alpha$ protein level determination. A thoracotomy was performed on the rats following sacrifice to obtain right and lower lung tissue samples. The samples were placed in a $-70^{\circ} \mathrm{C}$ refrigerator for the extraction of the total RNA and protein. BD-2 and TNF- $\alpha$ protein levels were detected using western blot analysis; the proteins were extracted with protein lysate (RIPA lysis buffer), diluted with $2 \mathrm{X}$ SDS buffer to the 
Table I. Comparison of the CK-MB and PPI values and BD-2 and TNF- $\alpha$ content levels with BD-2 mRNA expression in the four groups ( $\mathrm{n}=6 /$ group$)$.

\begin{tabular}{lcccrc}
\hline & \multicolumn{5}{c}{ Groups } \\
\cline { 2 - 5 } Indicators & $\mathrm{S}$ & $\mathrm{C}$ & $\mathrm{E}_{1}$ & $\mathrm{E}_{2}$ & F-value \\
\hline CK-MB (IU/l) & $13.667 \pm 8.477$ & $2847.167 \pm 408.040^{\mathrm{a}}$ & $1306.667 \pm 89.122^{\mathrm{a}, \mathrm{b}}$ & $1062.500 \pm 57.085^{\mathrm{a}-\mathrm{c}}$ & 184.715 \\
PPI $(\%)$ & $0.685 \pm 0.168$ & $4.287 \pm 1.778^{\mathrm{a}}$ & $2.780 \pm 0.604^{\mathrm{a}, \mathrm{b}}$ & $1.550 \pm 0.386^{\mathrm{a}-\mathrm{c}}$ & 30.456 \\
BD-2 protein & $0.292 \pm 0.088$ & $1.132 \pm 0.117^{\mathrm{a}}$ & $0.782 \pm 0.098^{\mathrm{a}, \mathrm{b}}$ & $0.613 \pm 0.083^{\mathrm{a}-\mathrm{c}}$ & 77.469 \\
TNF- $\alpha$ protein & $0.377 \pm 0.158$ & $1.127 \pm 0.024^{\mathrm{a}}$ & $0.918 \pm 0.163^{\mathrm{a}, \mathrm{b}}$ & $0.727 \pm 0.114^{\mathrm{a}-\mathrm{c}}$ & 37.392 \\
BD-2 mRNA & $0.402 \pm 0.697$ & $0.817 \pm 0.091^{\mathrm{a}}$ & $0.712 \pm 0.054^{\mathrm{a}, \mathrm{b}}$ & $0.622 \pm 0.044^{\mathrm{a}-\mathrm{c}}$ & 41.549
\end{tabular}

Results are expressed as the mean \pm standard deviation. ${ }^{a} \mathrm{P}<0.01$ compared with the $\mathrm{S}$ group; ${ }^{\mathrm{b}} \mathrm{P}<0.01$ compared with the $\mathrm{C}$ group; ${ }^{\mathrm{c}} \mathrm{P}<0.01$ compared with the $\mathrm{E}_{1}$ group. $\mathrm{CK}-\mathrm{MB}$, creatine kinase isoenzyme; PPI, lung permeability index; BD-2, $\beta$-defensin- 2 ; $\mathrm{S}$, sham operation group; $\mathrm{C}$, myocardial I/R control group; $\mathrm{E}_{1}$ and $\mathrm{E}_{2}$, edaravone-treated groups (different doses).

same concentration and boiled for $5 \mathrm{~min}$. The proteins were separated using SDS-PAGE gel electrophoresis and transferred to nitrocellulose (PVDF) membranes. The membranes were blocked overnight in a $5 \%$ bovine serum albumin (BSA) solution at $4^{\circ} \mathrm{C}$. For the primary antibody incubation, sc-47778 and sc-1348 were diluted at a ratio of 1:1,000 (TBST-diluted solution) and bs-1296R was diluted at a ratio of 1:200; these were maintained at $4^{\circ} \mathrm{C}$ overnight. Anti-mouse, anti-goat and anti-rabbit secondary antibodies were diluted at a ratio of 1:1,000 and incubated at room temperature for $2 \mathrm{~h}$. The grayscale value was used to determine BD- 2 and TNF- $\alpha$ protein expression following enhanced chemiluminescence (ECL) coloring and scanning.

$m R N A$ expression level determination. Total RNA was extracted from lung tissue samples (100 mg) using the TransZol one step method. $\beta$-actin was used as an internal standard to detect the BD-2 mRNA expression levels using a semi-quantitative RT-PCR assay. $\beta$-actin and BD-2 primers were designed in our laboratory and synthesized by Shanghai Sangon Biological Engineering Technology and Services Co., Ltd. (Shanghai, China). The primer sequences used were as follows: $\beta$-actin upstream, 5'-TGAACGGGAAGCTCACTGG-3' and downstream, 5'-TCCACCACCCTGTTGCTGTA-3' (product fragment length, 307 bp); and BD-2 upstream, 5'-TGCCTCCTTTTC TCCTATGC-3' and downstream, 5'-ATGGGAAACAGGTAC CCACA-3' (product fragment length, $216 \mathrm{bp})$. Total RNA (6 $\mu \mathrm{g})$ was used to clone cDNA with the TaqMan reverse transcription kit (Fermentas) and this was stored at $-20^{\circ} \mathrm{C}$. The PCR cycling conditions were as follows: $94^{\circ} \mathrm{C}$ for $20 \mathrm{sec}, 57^{\circ} \mathrm{C}$ for $30 \mathrm{sec}$ and $72^{\circ} \mathrm{C}$ for $30 \mathrm{sec}$ (36 cycles). RT-PCR products ( $\left.10 \mu \mathrm{l}\right)$ were used to carry out agarose gel electrophoresis for $2 \mathrm{~h}$ (voltage, $128 \mathrm{~V}$; current, $70 \mathrm{~mA}$; power, $9 \mathrm{~W}$ ), then stained with ethidium bromide. The RT-PCR amplification products were observed under an ultraviolet lamp, images were captured and an image analyzer was used to analyze the amplification products. The absorbance ratios of the target gene and $\beta$-actin were used to determine the BD-2 mRNA expression levels in the lung tissue.

Statistical analysis. SPSS 15.0 software was used for data analysis. Measurement data are expressed as the mean \pm stan- dard deviation (SD). The groups were compared using one-way ANOVA, and pairwise comparisons were performed using the SNK test $(\mathrm{a}=0.05)$. The BD-2 and TNF- $\alpha$ protein content of lung tissues in the $\mathrm{C}, \mathrm{E}_{1}$ and $\mathrm{E}_{2}$ groups were analyzed using Pearson's correlation analysis. $\mathrm{P}<0.05$ was considered to indicate a statistically significant difference.

\section{Results}

The CK-MB and PPI values of the $C, E_{1}$ and $E_{2}$ groups were significantly increased compared with those of the $\mathrm{S}$ group $(\mathrm{P}<0.01)$. Furthermore, the CK-MB and PPI values were decreased in the $E_{1}$ and $E_{2}$ groups compared with those of the $\mathrm{C}$ group and were also significantly decreased in the $\mathrm{E}_{2}$ group compared with those of the $\mathrm{E}_{1}$ group $(\mathrm{P}<0.01$; Table I).

A negligible level of BD-2 mRNA and BD-2 and TNF- $\alpha$ proteins were expressed in the $\mathrm{S}$ group; these indicators were significantly increased in the $\mathrm{C}, \mathrm{E}_{1}$ and $\mathrm{E}_{2}$ groups compared with the $\mathrm{S}$ group $(\mathrm{P}<0.01)$. The expression levels of BD-2 mRNA and BD-2 and TNF- $\alpha$ proteins in the $\mathrm{E}_{1}$ and $\mathrm{E}_{2}$ groups were decreased compared with those of the $\mathrm{C}$ group and were significantly decreased in the $\mathrm{E}_{2}$ group compared with those of the $\mathrm{E}_{1}$ group ( $\mathrm{P}<0.01$; Table I; Figs. 1 and 2).

In the $C, E_{1}$ and $E_{2}$ groups, the expression levels of BD-2 and TNF- $\alpha$ proteins were positively correlated with one another $(r=0.886, r=0.876$ and $r=0.878$, respectively; $\mathrm{P}<0.05$ for all the groups).

\section{Discussion}

The systemic immune response is often caused by lung, liver, heart and kidney I/R injury. The injury of these remote organs usually induces an acute immune response that is associated with white blood cell isolation and the release of enzymes from lung tissues. This increases vascular permeability, which causes perivascular and interstitial edema, leading to pulmonary hypertension and edema. Lung diseases caused by I/R injuries in these remote organs often lead to clinical respiratory distress syndromes (17).

In the present study, ligation of the LAD in rats for $45 \mathrm{~min}$ followed by relaxation for $3 \mathrm{~h}$ was applied to establish the 

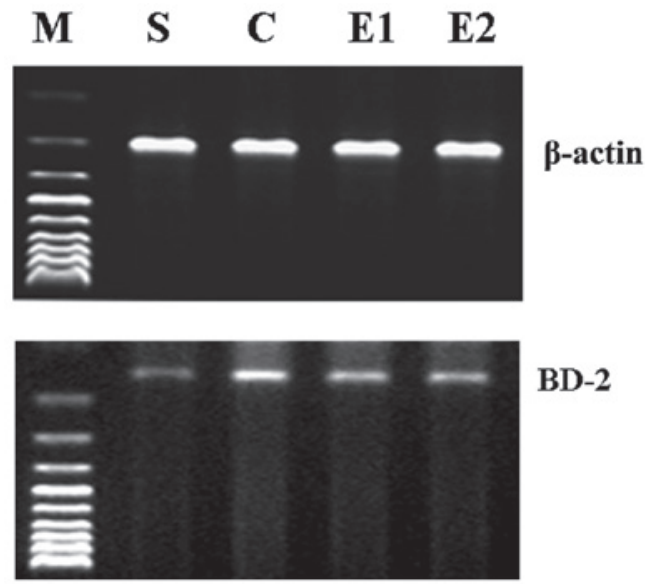

Figure 1. RT-PCR results for BD-2 mRNA expression in the 4 groups. $\mathrm{M}$, marker; $\mathrm{S}$, sham operation group; $\mathrm{C}$, myocardial $\mathrm{I} / \mathrm{R}$ control group; $\mathrm{E}_{1}$ and $\mathrm{E}_{2}$, edaravone-treated groups (different doses); BD-2, $\beta$-defensin-2.

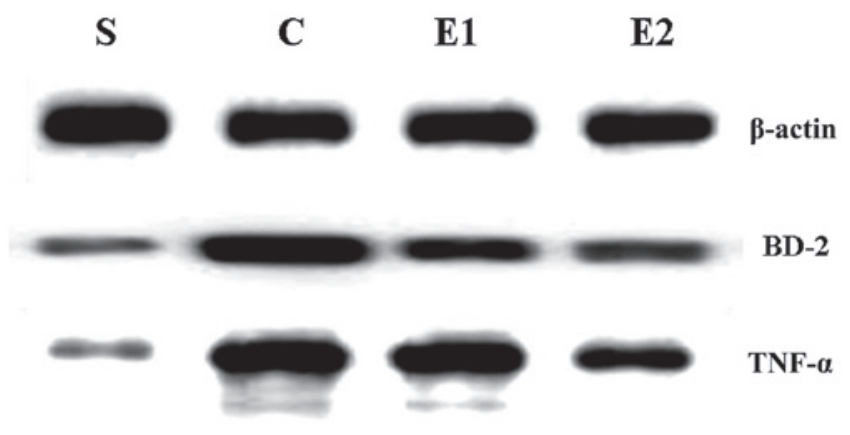

Figure 2. Western blotting results for BD-2 and TNF- $\alpha$ protein expression. $\mathrm{M}$, marker; $\mathrm{S}$, sham operation group; $\mathrm{C}$, myocardial I/R control group; $\mathrm{E}_{1}$ and $\mathrm{E}_{2}$, edaravone-treated groups (different doses); BD-2, $\beta$-defensin-2.

myocardial I/R model. During the experiment, elevation of the ST-segment in the lead-II occurred and the corresponding region on the heart surface darkened. The ligature was untied; when the electrocardiogram ST segment was depressed $>1 / 3$ and the heart surface had become its original red color, this indicated that the myocardial tissue had recovered from the reperfusion. At the end of the procedure, the serum CK-MB levels of the $C, E_{1}$ and $E_{2}$ groups were significantly increased. Since CK-MB is the most widely used enzyme for clinical diagnostic application in acute myocardial infarction (AMI), these increased levels indicated that the myocardial I/R model was successfully constructed in the present study.

The PPI indicates the permeability of lung tissue to proteins and lung I/R injury mainly appears to increase vascular permeability. The results of this study showed that the PPI values for the $C, E_{1}$ and $E_{2}$ groups were significantly higher compared with those of the $\mathrm{S}$ group, indicating that the permeability of the lung tissue increased after AMI and the presence of lung injury. These results are consistent with the results of previous studies (6), which demonstrates that construction of the acute myocardial I/R-induced lung injury model was successful.

Human BD-2 is mainly expressed in the skin, trachea and lung tissue. In foreskin-derived keratinocytes, BD-2 is rapidly expressed with the stimulation of TNF- $\alpha$ (within $1 \mathrm{~h}$ ) and this lasts for $>48 \mathrm{~h}$ (18). According to in vivo data, BD-2 protein levels in serum and BAL fluid are increased in cystic fibrosis and infectious lung disease patients (19). Alterations in the lung defense mechanisms of patients with chronic obstructive pulmonary diseases may promote the aggregation of bacteria in the peripheral trachea. Following examination, BD-2 expression levels in the peripheral trachea of these patients were increased. Patients who smoked exhibited decreased BD-2 secretion in the main tracheal epithelium, indicating that smoking may affect their condition through the alteration of lung tissue defense mechanisms (20). Following remote organ I/R injuries, numerous inflammatory cytokines are released by the lung tissue. For example, in intestinal I/R, TNF- $\alpha$, IL-6, IL-10, monocyte chemoattractant protein-1 (MCP-1) and interferon- $\gamma$ $(\mathrm{IFN}-\gamma)$ have been monitored in lung homogenates and BAL fluid samples (6). Ischemia of the mesenteric artery for $30 \mathrm{~min}$ caused gap inflammatory cell infiltration of the lung tissues and perivascular hemorrhagic edema occurred following reperfusion for $1 \mathrm{~h}$ (21). The results of the present study showed that the BD-2 mRNA level in the rat lung tissue was upregulated following myocardial I/R, accompanied by an increased expression of BD- 2 and TNF- $\alpha$ proteins, and the expression levels of these proteins were positively correlated. Therefore, considering the results of previous studies, we concluded that the release of numerous inflammatory mediators may induce the upregulation of BD-2 mRNA in lung tissue following myocardial I/R.

$\mathrm{BD}-2$ expression is regulated by downstream effectors (including the transcription factor NF- $\mathrm{\kappa B}$ or AP-1) and activated by excessive levels of proinflammatory cytokines (for example, IL-1 $\beta$, TNF- $\alpha$ and EGF). A number of studies have shown that NF- $\mathrm{KB}$ inhibitors significantly inhibit BD-2 mRNA upregulation; a possible explanation for this is that the binding sites of numerous transcription factors (including NF- $\kappa \mathrm{B}$ ) are the same as that of the BD-2 gene promoter (22). Following myocardial I/R injuries, numerous inflammatory cytokines (including TNF- $\alpha$ ) enter the lung tissue and further activate transcription factors, including NF-кB. These bind with cis-acting elements and then upregulate BD-2 gene expression at the transcription level (22).

Edaravone is a novel free radical scavenger, which has been shown to exert a protective effect in the I/R injuries of several organs. For example, in rabbit cardiomyocyte hypoxia-reoxygenation and heart I/R models, edaravone was shown to reduce cell damage and the myocardial infarction area during the reperfusion (oxygen) period $(23,24)$. A number of studies have reported that the use of edaravone reduced a variety of lung injuries, including in the in vitro lung I/R injury model (25), in acute lung injury caused by bleomycin (26) and in canine lung transplantation (27). This indicates that edaravone has the potential to have a protective effect in the donor lung ischemia protective solution and on lung receptors in lung transplantation reperfusion. Edaravone $(8 \mathrm{mg} / \mathrm{kg})$ has been shown to significantly reduce malondialdehyde levels in lung tissue following acute pancreatitis, inhibit neutrophil infiltration and lung tissue damage and reduce IL-6 and TNF- $\alpha$ levels (28).

In the present study, the CK-MB and PPI values of the two edaravone-treated groups were lower compared with those of the $\mathrm{C}$ group, indicating that damage to the heart and lungs was reduced. The levels of inflammatory cytokine TNF- $\alpha$ in these two groups were also decreased compared with the $\mathrm{C}$ group; similar to TNF- $\alpha$, BD-2 protein expression was reduced and 
BD-2 mRNA expression was downregulated. This showed that edaravone protects $\mathrm{I} / \mathrm{R}$ injury by inhibiting the release of proinflammatory cytokines, thus reducing the BD-2 gene and protein expression levels.

Edaravone exhibited dose-dependent protection in organ tissue damage. Nakamura et al (29) showed that of the 0.5-, 3-, 6- and $10-\mathrm{mg} / \mathrm{kg}$ doses of edaravone applied in a rat acute cerebral hemorrhage model, only 6 and $10 \mathrm{mg} / \mathrm{kg}$ had a protective effect on brain tissue. Edaravone has been shown to reduce the Fas-induced (Fas/CD95 is a cell surface protein belonging to the TNF receptor family) mortality in fulminant hepatic failure in a dose-dependent manner (30). Yuan et al (31) confirmed that edaravone used at an early stage with a high concentration in a rat Parkinson's disease model significantly reduced the degree of damage on autonomous behavior. In the present study, the use of $10 \mathrm{mg} / \mathrm{kg}$ edaravone caused CK-MB and PPI values to decrease to even lower levels compared with the decrease observed with $3 \mathrm{mg} / \mathrm{kg}$ edaravone. The expression levels of TNF- $\alpha$ and BD-2 proteins and BD-2 mRNA were also further reduced, indicating that edaravone decreased myocardial I/R lung tissue BD-2 gene and protein expression in a dose-dependent manner.

Due to time limitations, the present study did not investigate the time-dependent activity of edaravone in lung tissue. Different durations of treatment with various doses of edaravone may affect BD-2 expression. This requires investigation in further studies.

In conclusion, the BD-2 gene is upregulated in lung tissue following myocardial I/R and there was a significant inhibition of this upregulation of BD-2 after edaravone treatment; this occurred in a dose-dependent manner. This may have been caused by a reduction in TNF- $\alpha$ production, inhibiting the TNF- $\alpha$-induced BD-2 gene expression and resulting in the reduction of BD-2 mRNA and protein expression. However, the exact underlying mechanism remains to be fully elucidated.

\section{Acknowledgements}

This study was supported by the Funding Project 'Tackle Key Problems in Science and Technology' of the Provincial Health Bureau of Shanxi Province (No. 200948).

\section{References}

1. Murry CE, Jennings RB and Reimer KA: Preconditioning with ischemia: a delay of lethal cell injury in ischemic myocardium. Circulation 74: 1124-1136, 1986.

2. Mohan IK, Khan M, Wisel S, et al: Cardioprotection by HO-4038, a novel verapamil derivative, targeted against ischemia and reperfusion-mediated acute myocardial infarction. Am J Physiol Heart Circ Physiol 296: H140-H151, 2009.

3. Kutala VK, Khan M, Mandal R, et al: Attenuation of myocardial ischemia-reperfusion injury by trimetazidine derivatives functionalized with antioxidant properties. J Pharmacol Exp Ther 317: 921-928, 2006.

4. Tang YH, Xu JJ, Li JX and Cheng XS: Remote postconditioning induced by brief pulmonary ischemia and reperfusion attenuates myocardial reperfusion injury in rabbits. Chin Med J (Engl) 124 1683-1688, 2011.

5. Kitashiro S, Sugiura T, Tamura T, et al: Factors associated with increased extravascular lung water in cardiac tamponade and myocardial ischemia. Crit Care Med 27: 2229-2233, 1999.

6. He X, Han B, Mura M, et al: Anti-human tissue factor antibody ameliorated intestinal ischemia reperfusion-induced acute lung injury in human tissue factor knock-in mice. PLoS One 3: e1527, 2008 .
7. Faubel S: Pulmonary complications after acute kidney injury. Adv Chronic Kidney Dis 15: 284-296, 2008.

8. Andrés-Hernando A, Altmann C, Ahuja N, et al: Splenectomy exacerbates lung injury after ischemic acute kidney injury in mice. Am J Physiol Renal Physiol 301: F907-F916, 2011.

9. Shu Q, Shi Z, Zhao Z, et al: Protection against Pseudomonas aeruginosa pneumonia and sepsis-induced lung injury by overexpression of beta-defensin-2 in rats. Shock 26: 365-371, 2006.

10. Hu Q, Zuo P, Shao B, et al: Administration of nonviral gene vector encoding rat beta-defensin-2 ameliorates chronic Pseudomonas aeruginosa lung infection in rats. J Gene Med 12: 276-286, 2010.

11. Wu QP, Yao SL and Fang XM: Study of rat beta-defensin-2 gene and protein expression in ventilator-associated pneumonia. Zhongguo Wei Zhong Bing Ji Jiu Yi Xue 17: 353-356, 2005 (In Chinese).

12. Liu KX, Chen SQ, Zhang H, et al: Intestinal ischemia/reperfusion upregulates beta-defensin-2 expression and causes acute lung injury in the rat. Injury 40: 950-955, 2009.

13. Yamazaki K, Miwa S, Toyokuni S, et al: Effect of edaravone, a novel free radical scavenger, supplemented to cardioplegia on myocardial function after cardioplegic arrest: in vitro study of isolated rat heart. Heart Vessels 24: 228-235, 2009.

14. Liu N, Shang J, Tian F, et al: In vivo optical imaging for evaluating the efficacy of edaravone after transient cerebral ischemia in mice. Brain Res 1397: 66-75, 2011.

15. Shimoda M, Iwasaki Y, Okada T, et al: Edaravone inhibits apoptosis caused by ischemia/reperfusion injury in a porcine hepatectomy model. World J Gastroenterol 18: 3520-3526, 2012.

16. Salvi S: Protecting the myocardium from ischemic injury: a critical role for alpha(1)-adrenoreceptors? Chest 119: 1242-1249, 2001

17. Waisman D, Brod V, Dickstein R, et al: Effects of inhaled nitric oxide on lung injury after intestinal ischemia-reperfusion in rats. Shock 23: 150-155, 2005.

18. Chung WO and Dale BA: Innate immune response of oral and foreskin keratinocytes: utilization of different signaling pathways by various bacterial species. Infect Immun 72: 352-358, 2004.

19. van Wetering S, Sterk PJ, Rabe KF and Hiemstra PS: Defensins: key players or bystanders in infection, injury, and repair in the lung? J Allergy Clin Immunol 104: 1131-1138, 1999.

20. Pace E, Ferraro M, Minervini MI, et al: Beta defensin-2 is reduced in central but not in distal airways of smoker COPD patients. PLoS One 7: e33601, 2012.

21. Onder A, Kapan M, Gümüs M, et al: The protective effects of curcumin on intestine and remote organs against mesenteric ischemia/reperfusion injury. Turk J Gastroenterol 23: 141-147, 2012.

22. Steubesand N, Kiehne K, Brunke G, et al: The expression of the beta-defensins hBD-2 and hBD-3 is differentially regulated by NF-kappaB and MAPK/AP-1 pathways in an in vitro model of Candida esophagitis. BMC Immunol 10: 36, 2009.

23. Yamawaki M, Sasaki N, Shimoyama M, et al: Protective effect of edaravone against hypoxia-reoxygenation injury in rabbit cardiomyocytes. Br J Pharmacol 142: 618-626, 2004.

24. Wu TW, Zeng LH, Wu J and Fung KP: Myocardial protection of MCI-186 in rabbit ischemia-reperfusion. Life Sci 71: 2249-2255, 2002.

25. Reyes YA, Shimoyama T, Akamatsu H and Sunamori M: MCI-186 (edaravone), a free radical scavenger, attenuates ischemia-reperfusion injury and activation of phospholipase $\mathrm{A}(2)$ in an isolated rat lung model after $18 \mathrm{~h}$ of cold preservation. Eur J Cardiothorac Surg 29: 304-311, 2006.

26. Asai T, Ohno Y, Minatoguchi S, et al: The specific free radical scavenger edaravone suppresses bleomycin-induced acute pulmonary injury in rabbits. Clin Exp Pharmacol Physiol 34: 22-26, 2007.

27. Xu JZ, Shen BZ, Li Y, et al: Edaravone attenuates ischemia-reperfusion injury by inhibiting oxidative stress in a canine lung transplantation model. Chin Med J (Engl) 121: 1583-1587, 2008.

28. Yang T, Mao YF, Liu SQ, et al: Protective effects of the free radical scavenger edaravone on acute pancreatitis-associated lung injury. Eur J Pharmacol 630: 152-157, 2010.

29. Nakamura T, Kuroda Y, Yamashita S, et al: Edaravone attenuates brain edama and neurologic deficits in a rat model of acute intracerebral hemorrhage. Stroke 39: 463-469, 2008.

30. Miyasou T, Kwon AH, Tsuji K, et al: Edaravone prevents Fas-induced fulminant hepatic failure in mice by regulating mitochondrial Bcl-xL and Bax. Shock 30: 212-216, 2008.

31. Yuan WJ, Yasuhara T, Shingo T, et al: Neuroprotective effects of edaravone-administration on 6-OHDA-treated dopaminergic neurons. BMC Neurosci 9: 75, 2008. 